\title{
Improving access to urologists through an electronic consultation service
}

\author{
Luke Witherspoon, MD, MB, MSc, BSC '; Clare Liddy, MD, FCFPC'; Amir Afkham, MSc ${ }^{3}$; Erin Keely, MD, FRCPC ; \\ John Mahoney, MSC, MD, FRCSC ${ }^{1}$
}

'Department of Urology, University of Ottawa; ${ }^{2}$ Department of Family Medicine, University of Ottawa and C.T. Lamont Primary Health Care Research Centre, Bruyère Research Institute; ${ }^{3}$ Champlain LHIN; ${ }^{4}$ Department of Medicine, University of Ottawa and Ottawa Hospital Research Institute; Ottawa, ON, Canada

Cite as: Can Urol Assoc J 2017; 11 (8):270-4. http://dx.doi.org/10.5489/cuaj.4314

\section{Abstract}

Introduction: Access to specialist services is limited by wait times and geographic availability. Champlain Building Access to Specialist Advice (BASE) has been implemented in our service region to facilitate access to specialists by primary care providers (PCPs). Through a secure web-based system, PCPs are able to send eConsults instead of requesting a formal in-office consultation.

Methods: Urology eConsults completed through the Champlain BASE service from March 2013 to January 2015 were analyzed. Each consult was characterized in regard to the type of question asked by the referring physician and the clinical content of the referral. Using the mandatory close-out surveys, we analyzed rates of referral avoidance, physician satisfaction, and overall impact on patient care.

Results: Of 190 eConsultations, 70\% were completed in less than 10 minutes. The most common clinical questions related to the interpretation of imaging reports $(16 \%)$ and tests to choose for investigating a condition (15\%). The most common diagnoses were hematuria (13\%) and renal mass ( $8 \%$ ). In $35 \%$ of cases, referral to a urologist had originally been contemplated and was avoided. In $8 \%$ of cases, a PCP did not believe a consultation was initially needed, but a referral was ultimately initiated after the eConsultation.

Conclusions: Our study shows that although certain clinical presentations still require a formal in-person urological consultation, eConsultations can potentially reduce unnecessary clinic visits while identifying patients who may benefit from early urological consultation. Through both these mechanisms, we may improve timely access to urologists.

\section{Introduction}

Access to surgical specialists is projected to become an increasingly difficult problem in North America. ${ }^{1-4}$ Already within Canada, the average wait time to be referred to a urologist is 10.8 weeks, and the average time to treatment once seeing a urologist is 5.4 weeks. ${ }^{5}$ These wait times are in discordance with the specialty's own views, with a survey of urological specialists concerning a "clinically reasonable" wait time revealing that urologists in Canada viewed a wait time of 4.2 weeks as "clinically reasonable." ${ }^{5}$

Long wait times can lead to late diagnosis and poor patient outcomes. One study from England examined 55 patients who had been triaged as non-urgent and who would have otherwise waited for an average of 13 weeks to see a urologist and found that seven of the 55 patients ultimately went on to be diagnosed with a urological malignancy relating to their initial complaint. ${ }^{6}$ Furthermore, a Canadian study found that longer wait times increased the overall mortality of patients diagnosed with bladder cancer. ${ }^{7}$

To help reduce long wait times, innovative technologies must be incorporated into our practice to improve access to specialists. Virtual consultation, either through telemedicine, which uses technology to provide clinical healthcare to a patient from a distance, or electronic consultation (eConsults), which provides a communication channel for providers to asynchronously discuss a clinical case, are used to replace the need for a face-to-face visit. Use of telemedicine in urology has been reported in a few small studies from the U.S. Patients are willing to receive urological care through telemedicine and do not view the practice negatively. ${ }^{8}$ Urological telemedicine has been studied in a small number of pediatric populations and has been found to provide better continuity of care by eliminating distance barriers and reducing the burden on families travelling to bring their children to more traditional appointments. ${ }^{9,10}$ Studies in the adult population have so far been mainly limited to the application of remote telesurgery, with the advent of robotic surgical systems. ${ }^{11,12}$ Some small, single-centre studies of the adult urological population and telemedicine in the U.S. have so far shown good patient/provider satisfaction and success. ${ }^{13-16}$

There are no reports of using provider-to-provider eConsults in urology. A systematic review of 27 U.S.-based papers published on eConsultations showed that eConsults were widely applicable across several different specialties and 
they generally reduced the need for face-to-face visits, while providing highly satisfactory results to the primary care providers (PCPs) involved. ${ }^{17,18}$

The eConsult service used in our study was the Champlain Building Access to Specialists through eConsultation (BASE) service developed in Ottawa, Canada. It has been available since 2010and now has 1101 PCPs registered who can access 93 specialty services, including urology. As of November 1, 2016, over 18000 eConsults have been completed. ${ }^{19-21}$

The purpose of this study is to describe the use and impact of the eConsult service in urology and to characterize the type and content of clinical questions being asked. Our hypothesis is that the use of an eConsult service will help reduce the need for in-person urological consultation.

\section{Methods}

\section{Champlain BASE eConsult service}

Studies using the Champlain BASE system that detail its process have been published previously. ${ }^{22}$

The Champlain BASE system is a secure, web-based service that interfaces between a PCP (general practitioner or nurse practitioner) and a specialist. The PCP is able to submit questions using a standardized, patient-specific form. Other information (laboratory results, medical imaging, etc.) can be attached as is pertinent to the question being asked. The receiving specialist can then provide advice concerning further required investigations, potential treatment options, or provide a recommendation for a face-to-face consultation.

Before each encounter between a PCP and specialist can be closed in the system, the PCP completes a mandatory survey. This survey allows the PCPs to rank their experience with this service and provide feedback on how useful they found the referral to be. This close-out survey further assesses the impact on a PCP electing to refer on to see a specialist in person.

The end of service survey contains five sections:

1. Allows the PCP to choose from four options concerning how the eConsult affected the outcome for the patient.

2. Allows PCP to choose from six options concerning how the interaction affected his/her decision to refer or not.

3. Ranks eConsult's value for the patient on a five-point Likert scale.

4. Ranks eConsult's value for the physician on a fivepoint Likert scale.

5. Free text space for additional comments.

\section{Study participants}

One-hundred and ninety eConsults directed to urology and completed through the Champlain BASE service from March 2013 to January 2015 were analyzed. The patient population encompassed in this study is the Champlain region of Eastern Ontario, which has approximately 1.2 million citizens, serviced by one tertiary care hospital with three campuses.

\section{Data collection}

All data presented in this study was collected, stored securely, and then retrospectively accessed for analysis. Data available for analysis included type of PCP, the length of time for the PCP to receive the specialist reply, time required for the specialist to complete the eConsult, and the PCP response to the end of interaction survey.

\section{Methods of question categorization}

All eConsults within the defined study period were reviewed by a single rater and categorized retrospectively by clinical content and type of question asked by the PCP. A predefined list of clinical topics (based on ICPC-2 taxonomy and refined by consensus) and types of questions (based on validated taxonomy) were created to facilitate the eConsult review and categorization..$^{23} \mathrm{~A}$ total of 24 clinical content and 10 types of clinical questions were used in the categorization.

\section{Results}

From March 2013 to January 2015, 190 consultations were directed to urology through the Champlain BASE system and answered by two urologists. Of the 190 eConsults, 174 were from other physicians (91.6\%) and $16(8.4 \%)$ were from nurse practitioners. The average time for a urologist to respond to a PCP's question was 2.51 days, with a median of 0.92 days and range of 11 minutes to 18 days.

Self-reported time for a consulting urologist to complete the eConsult was fewer than 10 minutes in $70 \%$ of cases, $15-20$ minutes in $15 \%$ of cases, $10-15$ minutes in $10 \%$ of cases, and greater than 20 minutes in $5 \%$ of cases.

Overall, PCPs rated the service very highly, with $89 \%$ giving a rating of 4 or 5 on a five-point Likert scale for value to the patient (Fig. 1). Similarly, $90 \%$ of PCPs gave the service a rating of 4 or 5 on the same Likert scale for its overall value for the healthcare provider (Fig. 2).

Two questions from the survey identified a change in physician behaviour. In $35 \%$ of cases, the PCP had intended to refer the patient for an in-office consultation, but no longer needed to after receiving advice through the eConsult (referral avoided). Thirty-seven percent of eConsults were found to result in an in-office visit still being needed; $17 \%$ were 


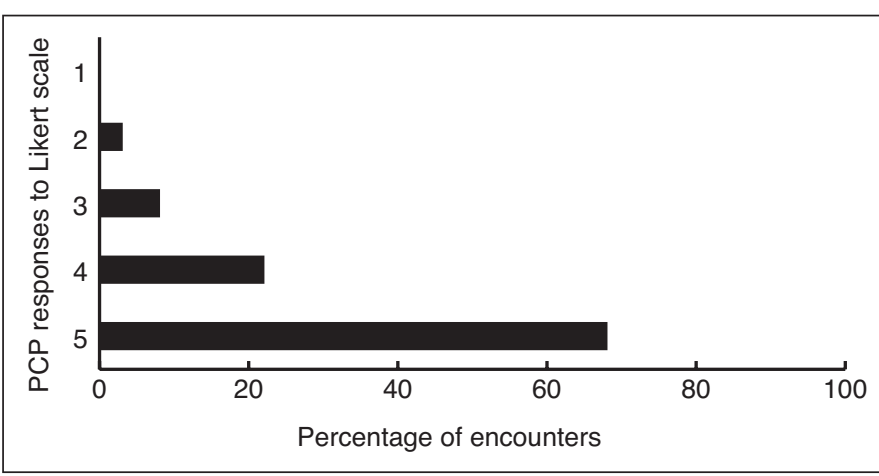

Fig. 1. eConsultation effect on patient ranked by primary care providers (PCPs). Five-point Likert scale was used, with $1=$ minimal and $5=$ excellent.

initially thought by the PCP to not need a referral and after the interaction were still found to not need an in-office visit with a urologist. Interestingly, in $8 \%$ of eConsults, a new referral was identified as needed where the PCP had not initially thought one was needed. In $2 \%$ of eConsults, the PCP found the service to be of no benefit (Fig. 3). In $62 \%$ of eConsultations, a new course of action was taken as a result of the interaction.

The clinical content of the eConsults revealed a diverse set of clinical content areas and patient symptoms. Some of the most common topics were hematuria $(13 \%)$, renal masses $(7.9 \%)$, kidney stones $(7.3 \%)$, and urinary tract infections (6.3\%) (Fig. 4). With regards to general question categories, interpretation of an image report was the most common (16.3\%). Following this were: what test to choose to further investigate a symptom $(14.7 \%)$, interpretation of a clinical finding $(14.2 \%)$, and medication-related questions (13.1\%) (Fig. 5).

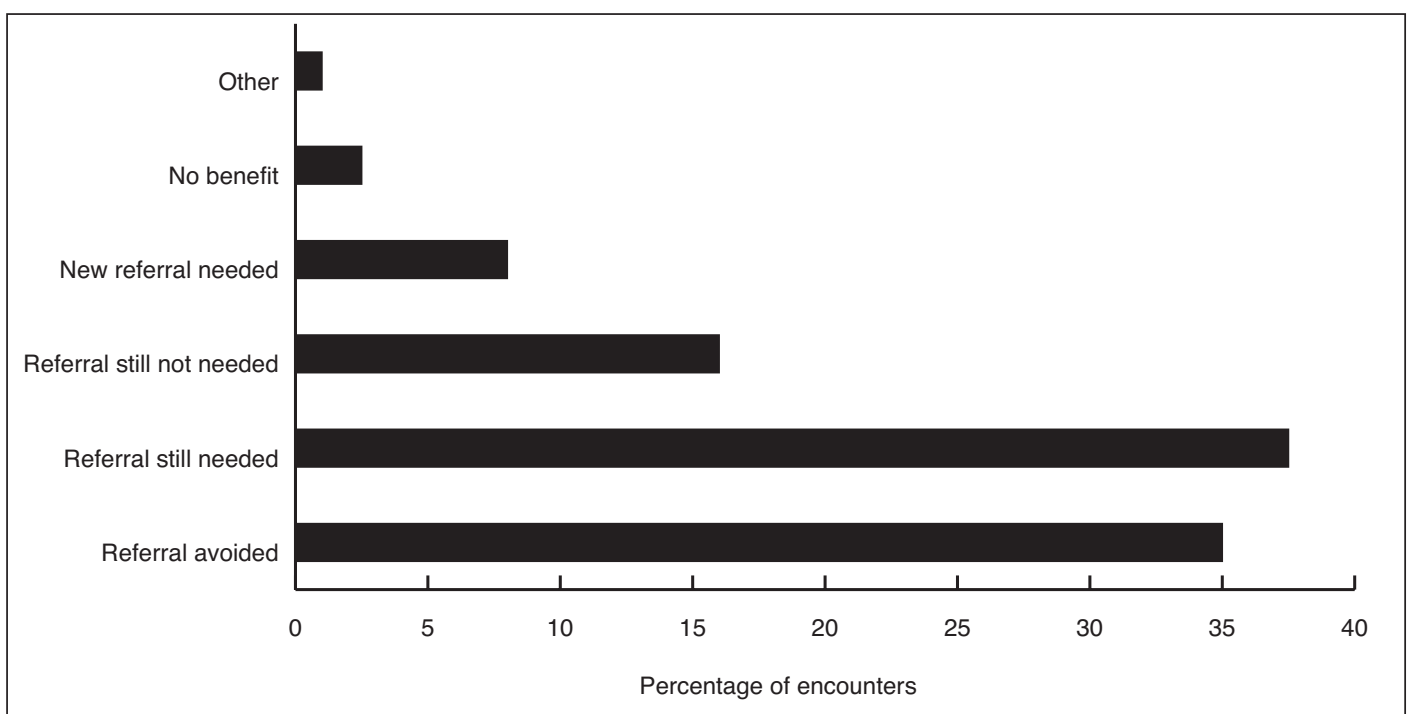

Fig. 3. Outcomes following completion of eConsultation.

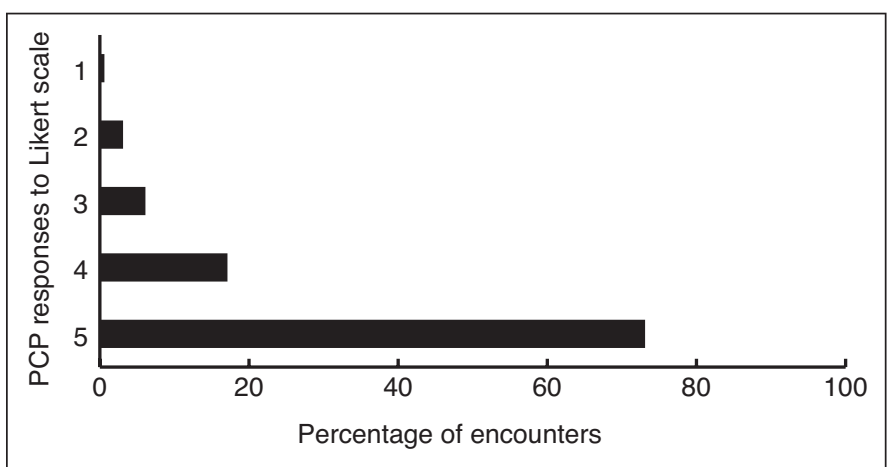

Fig. 2. eConsultation usefulness for primary care providers (PCPs) ranked by PCPs. Five-point Likert scale was used, with $1=$ minimal and $5=$ excellent.

\section{Discussion}

This study is the first to report on the use of eConsults in the specialty of urology. The referral "avoidance" rate of 35\% potentially represents a sizable proportion of patients who are currently being referred to urologists, but who do not actually require an in-office visit. Despite being based on a small sample population, this referral avoidance rate is comparable to other services offered through Champlain BASE and other large eConsult services..$^{24-26}$ Being able to decrease in-office visits by a third would have significant effects on wait times, allowing those who need in-office consultations to access care more quickly. Further adding to the safety aspect of this service, we found that $8 \%$ of patients were found to require an in-office visit, although the PCP did not initially think this was necessary. This suggests the service not only helps prevent unneeded consultation, but allows identification of patients who may otherwise have delayed specialist care, as their symptoms had not been identified as requiring a specialist appointment by their PCP.

Our results our similar to what has been reported in other specialties in Champlain BASE and other eReferral/eConsult studies examining preconsultation exchanges between PCP and specialists. $^{24-27}$ These studies have shown similar numbers of referrals that did not actually need to be seen by specialists. This is important, as it helps to allay some of the fear that patients are being missed by not being physically 


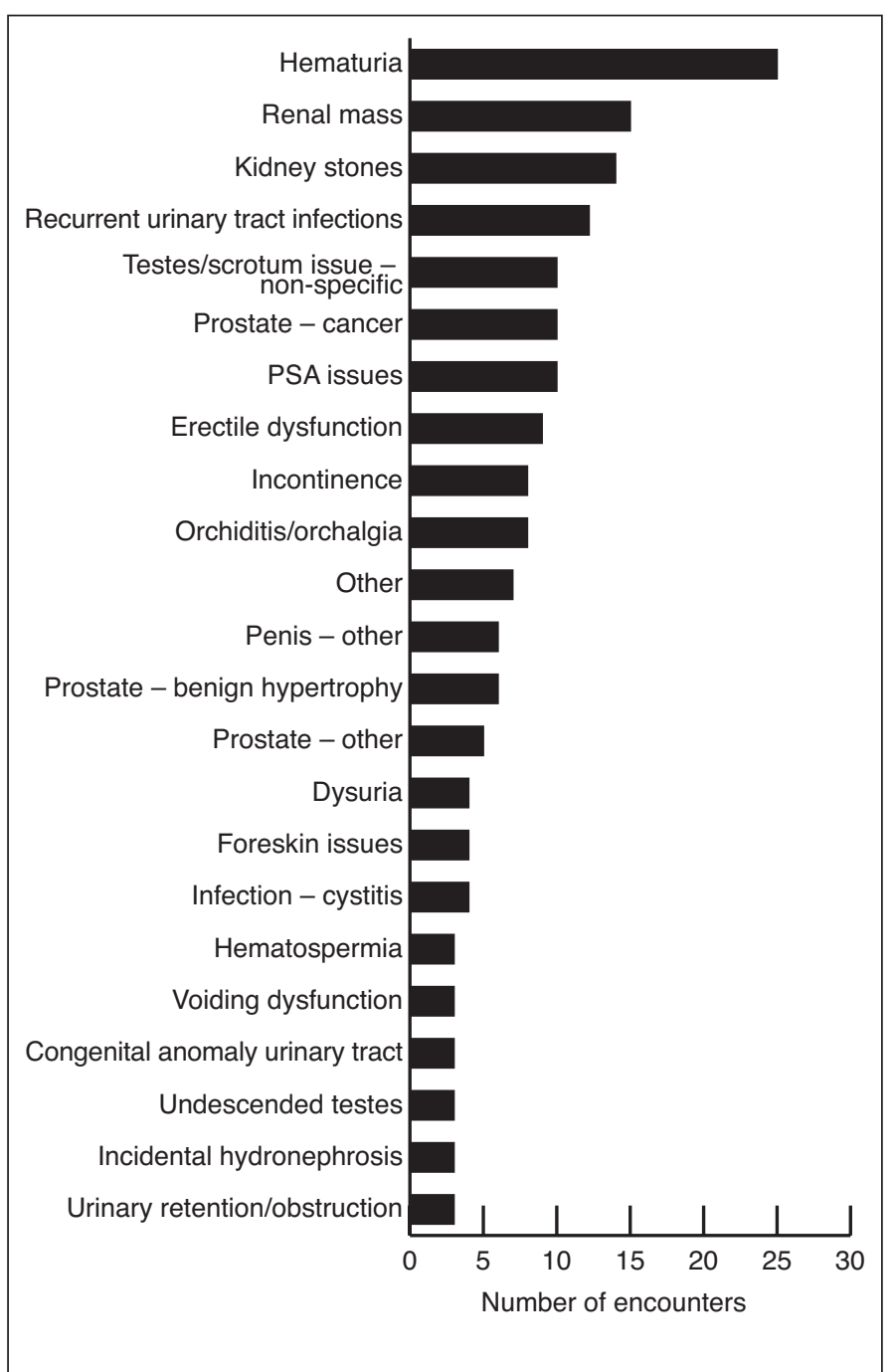

Fig. 4. Topics of eConsultations.

seen, as multiple studies across specialties consistently show eConsult to be an evolving and safe method of triaging patient referrals.

Providing eConsults is not time-intensive. Most are answered in less than 10 minutes. The benefits to the participating urologists include the unique opportunity for feedback directly from the PCP through the mandatory survey. This provides a unique system of physician-to-physician feedback, which is lost in the traditional referral-based system. As an added value, specialists would be able to track their strengths and weaknesses within the framework of the eConsultation system if they so desire.

Review of the common question topics and subjects asked through the service provides insight into the real-time clinical challenges for PCPs. This has the potential to inform the planning of continuing medical education (CME) events for primary care providers and potentially affecting curriculums of medical and nursing schools if areas of medical knowledge are consistently identified as knowledge gaps.

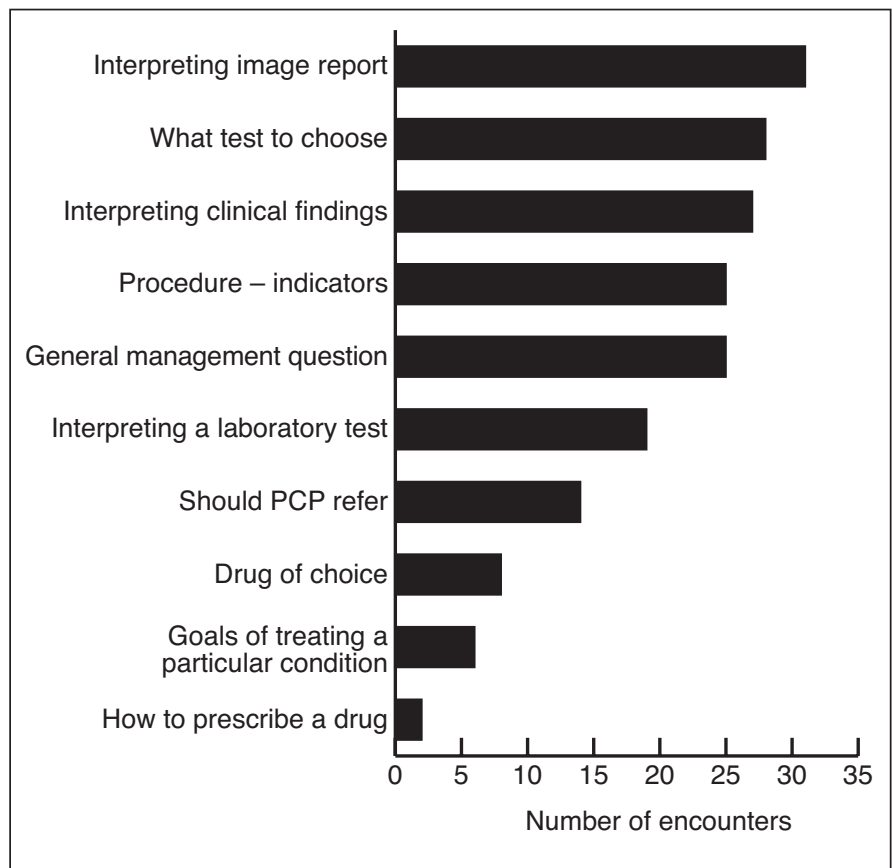

Fig. 5. Question categories posed by primary care providers.

\section{Study limitations}

This study does have limitations to consider. First, this is a small sample size of only 190 patients from one service. Furthermore, we do not have any long-term followup and were unable to see whether any of the eConsults ending in referral avoidance ultimately did require an in-office visit. Instead, we are left relying on the end of consultation survey done by the PCP. In addition, we are unable to track adverse events occurring from the eConsult service and so are not able to directly assess the safety of such a system.

\section{Conclusion}

eConsultations are an effective tool for the field of urology to continue to explore. With the potential to significantly reduce in-office visits, adopting technologies such as an eConsult service represents a unique avenue to improve access to urology services and strengthen communication with primary care communities.

Competing interests: The authors report no competing personal or financial interests.

Acknowledgement: This work was supported by the Ministry of Health and Long-Term Care, The Canadian Institutes of Health Research, The Ottawa Hospital Academic Medical Organization Innovation Fund, e-Health Ontario, the University of Ottawa Department of Medicine, Bruyère Research institute, and the Champlain Local Health Integration Network.

This paper has been peer-reviewed. 


\section{References}

1. Wei J, Calhoun E, Jacobsen S. Urologic diseases in America project: Benign prostatic hyperplasia. J Urol 2005;173:1256-61. https://doi.org/10.1097/01.ju.0000155709.37840.fe

2. Aytac I, Mckinlay J, Krane R. The likely worldwide increase in erectile dysfunction between 1995 and 2025 and some possible policy consequences. BJU Int 1999;84:50-6. https://doi.org/10.1046/j.1464410x.1999.00142.x

3. Etzioni $\mathrm{D}$, Liu J, Maggard $\mathrm{M}$, et al. The aging population and its impact on the surgery workforce. Ann Surg 2003;238:170-7. htrps://doi.org/10.1097/01.SLA.0000081085.98792.3d

4. Schulman C. The aging male: A challenge for urologists. Curr Opin Urol 2000;10:337-42. https://doi.org/10.1097/00042307-200007000-00008

5. Fraserinstitute.org. Toronto. Waiting your turn: Wait times for healthcare in Canada, 2016 Report; 22014 [updated November 23 2016]. Available at htrps://www.fraserinstitute.org/sites/default/files/waitingyour-turn-2015.pdf. Accessed November 24, 2016.

6. German K, Nuwahid F, Matthews $P$, et al. Dangers of long waiting times for outpatient appointments at a urology clinic. BMJ 1993;306:429. htrps://doi.org/10.1136/bmi.306.6875.429

7. Kulkarni $G$, Urbach D, Austin $P$, et al. Longer wait times increase overall mortality in patients with bladder cancer. J Urol 2009;182:1318- 24. https://doi.org/10.1016/i.juro.2009.06.041

8. Viers $B$, Pruthi $S$, Rivera $M$, et al. Are patients willing to engage in telemedicine for their care: A survey of pre-use perceptions and acceptance of remote video visits in a urological patient population. Urology 2015;85:1233-40. https://doi.org/10.1016/i.urology.2014.12.064

9. Canon S, Shera A, Patel A, et al. A pilot study of telemedicine for postoperative urological care in children. J Telemed Telecare 2014;20:427-30. https://doi.org/10.1177/1357633X14555610

10. Weinberg AE, Costaglio C, Pike S. Innovations in pediatric urology telehealth: Changing the landscape of surgical care in the digital age. J Urol 2014;191:62. https://doi.org/10.1016/i.juro.2014.02.248

11. Nguan C, Morady R, Wang C, et al. Robotic pyeloplasty using internet protocol and satellite network-based telesurgery. Int J Med Robot 2008;4:10-4. hittps://doi.org/10.1002/rss. 173

12. Sterbis J, Hanly E, Herman B, et al. Transcontinental telesurgical nephrectomy using the da Vinci robot in a porcine model. Urology 2008;71:971-3. https://doi.org/10.1016/i.urology.2007.11.027

13. Hayes $W$, Tohme W, Komo D, et al. A telemedicine consultative service for the evaluation of patients with urolithiasis. Urology 1998:51:39-43. https://doi.org/10.1016/S0090-4295(97)00486-X

14. Liatsikos EN, Gortzis L, Nikiforidis $G$, et al. Telediagnostic and therapeutic guidance in urology. J Endourol 2004;18:625-8. htrps://doi.org/10.1089/end.2004.18.625

15. Challacombe B, Kavoussi L, Patriciu A. Technology insight: Telementoring and telesurgery in urology. Nat Rev Urol 2006;3:611-7. https://doi.org/10.1038/ncpuro0626

16. Park E, Boedeker B, Hemstreet J, et al. The initiation of a preoperative and postoperative telemedicine urology clinic. Stud Health Technol Inform 2010;163:425-7.
17. Vimalananda V, Gupte G, Serai SM, et al. Electronic consultations (eConsults) to improve access to specialty care: A systematic review and narrative synthesis. J Telemed Telecare 2015;21:323-30. https://doi.org/10.1177/1357633X15582108

18. Liddy C, Drosinis P, Keely E. Electronic consultation systems: Worldwide prevalence and their impact on patient care — a systematic review. Fam Pract 2016;33:274-85. https://doi.org/10.1093/ fampra/cmw024

19. Keely E, Drosinis P, Afkham A, et al. Perspectives of Champlain BASE specialist physicians: Their motivation, experiences, and recommendations for providing eConsultations to primary care providers. Stud Health Technol Inform 2015;209: 38-45.

20. Liddy C, Afkham A, Drosinis P, et al. Impact of and satisfaction with a new eConsult service: A mixed methods study of primary care providers. J Am Board Fam Med 2015;28:394-403. https://doi.org/10.3122/jabfm.2015.03.140255

21. champlainbaseeconsult.com. Ottawa. Champlain BASE eConsult. CC [updated 2016 Sep 14]. Available at www.champlainbaseeconsult.com. Accessed October 10, 2016.

22. Keely E, Liddy C, Afkham A. Utilization, benefits, and impact of an eConsultation service across diverse specialties and primary care providers. Telemed E-Health 2013; 19:733-8. https://doi.org/10.1089/ tmi.2013.0007

23. Ely J, Osheroff J, Gorman P, et al. A taxonomy of generic clinical questions: Classification study. BMJ 2000;321:429-432. htrps://doi.org/10.1136/bmi.321.7258.429

24. Fogel A, Khamisa K, Afkham A, et al. Ask the eConsultant: Improving access to hematology expertise using an asynchronous eConsult system. J Telemed Telecare 2017;23:421-42. https://doi.org/10.1177/1357633X16644095

25. Liddy C, Smyth C, Poulin P, et al. Improving access to chronic pain services through eConsultation: A cross-sectional study of the Champlain BASE eConsult service. Pain Med 2016. Epub ahead of print. https://doi.org/10.1093/pm/pnw038

26. Keely E, Liddy C, Afkham A. Utilization, benefits, and impact of an eConsultation service across diverse specialties and primary care providers. Telemed E-Health 2013;19:733-8.

27. Shehata $F$, Posner $G$, Afkham $A$, et al. Evaluation of an electronic consultation service in obstetrics and gynecology in Ontario. Obstet Gynecol 2016;127:1033-8. https://doi.org/10.1097/ A0G.0000000000001431

Correspondence: Dr. John Mahoney, Department of Urology, University of Ottawa, Ottawa, ON; jomahoney@toh.ca 